\title{
An expectations-based approach to explaining the cross-modal influence of color on orthonasal olfactory identification: The influence of the degree of discrepancy
}

\author{
MaYa Shankar \\ University of Oxford, Oxford, England \\ Christopher Simons \\ Givaudan, Cincinnati, Ohio \\ Baba Shiv and Samuel McClure \\ Stanford University, Stanford, California \\ Carmel A. LeVitan \\ Occidental College, Los Angeles, California \\ AND \\ Charles Spence \\ University of Oxford, Oxford, England
}

\begin{abstract}
In the present study, we explored the conditions under which color-generated expectations influence participants' identification of flavored drinks. Four experiments were conducted in which the degree of discrepancy between the expected identity of a flavor (derived from the color of a drink) and the actual identity of the flavor (derived from orthonasal olfactory cues) was examined. Using a novel experimental approach that controlled for individual differences in color-flavor associations, we first measured the flavor expectations held by each individual and only then examined whether the same individual's identification responses were influenced by his or her own expectations. Under conditions of low discrepancy, the perceived disparity between the expected and the actual flavor identities was small. When a particular color-identified by participants as one that generated a strong flavor expectation - was added to these drinks (as compared with when no such color was added), a significantly greater proportion of identification responses were consistent with this expectation. This held true even when participants were explicitly told that color would be an uninformative cue and were given as much time as desired to complete the task. By contrast, under conditions of high discrepancy, adding the same colors to the drinks no longer had the same effect on participants' identification responses. Critically, there was a significant difference in the proportion of responses that were consistent with participants' color-based expectations in conditions of low as compared with high discrepancy, indicating that the degree of discrepancy between an individual's actual and expected experience can significantly affect the extent to which color influences judgments of flavor identity.
\end{abstract}

As we interact with the world around us, we are continually met by a number of distinct and salient smells: the waft of buttered popcorn that strikes us when we first enter the movie theater, the unpleasant smell of milk that has soured after being left out a bit too long, and the cup of freshly roasted coffee that sits at our desk, helping to keep us attentive during the day. Our sense of smell is undoubtedly advantageous from an evolutionary point of view. Being able to perceive and recognize the odors in our environment can provide useful information: Smells can help us to avoid a burning building to our right, to consume a nutritious piece of fruit over a rotten one, and to help us select a fitting mate (see Candolin, 2003; Desor \& Beauchamp, 1974; Rabin \& Cain, 1984; Stevenson, 2009; Vlahos, 2006).

Interestingly, though, our ability to correctly identify substances just on the basis of orthonasal olfactory cues is actually quite poor. When odor cues are presented in isolation (i.e., divorced from any context), we are able to correctly identify only roughly one third to one half of them (see Cain, 1979; Engen \& Ross, 1973; Rabin \&

M.Shankar, mayas@stanford.edu 
Cain, 1984; Richardson \& Zucco, 1989; Schab, 1991). And, although we are certainly better at identifying more familiar odors than less familiar ones, it has been reported that we only perform with slightly more accuracy $(5 \%)$ in these tasks (see Zellner, Bartoli, \& Eckard, 1991, for a review).

These results are perhaps not so surprising when one considers the fact that under more natural conditions, we rarely have to identify a substance without access to a wealth of additional information from both higher level cognition and other sensory modalities. For example, we are usually able to view the food or drink that we happen to smell or, in more ambiguous settings (i.e., when the foodstuff is heavily packaged), are able to read packaging labels that typically provide useful information regarding the foodstuff's identity. Sometimes, contextual information of this kind is actually imperative for the correct identification of an object: Parmesan cheese and vomit, for example, have olfactory components with similar bases (isovaleric and butyric acid). Consequently, this acid can be perceived as either of these two identities equally well (see Herz \& von Clef, 2001). Without the assistance of visual cues to set up an expectation as to the likely identity of the substance in question, we can imagine accidentally throwing out a perfectly good meal because it is mistaken for something quite different!

Only recently have scientists discovered that our perceptions and judgments of information in one sense (e.g., olfaction) are actually quite susceptible to the influence of information from the other senses (e.g., vision). Crossmodal influences of this kind can help us to more successfully identify the objects in our external world (see, e.g., Cardello, 2007, for a review). Given that we rarely receive olfactory cues in isolation, but instead do so against a much richer contextual backdrop, our olfactory judgments of a drink's flavor are often prone to the effects of expectations derived from these other sensory sources (see Auvray \& Spence, 2008; Stevenson, 2009; Verhagen \& Engelen, 2006, for recent reviews of multisensory flavor perception).

\section{The Influence of Color on Orthonasal Odor Identification}

One such source of information concerning odor identity comes from color (see, e.g., Hutchings, 2003). Cardello (1994) pointed out that visual cues are likely to generate salient expectations about a food or drink's characteristics since they often convey the first sensory impressions of that stimulus to the perceiver (see also Masurovsky, 1939). Color cues, in particular, seem likely to generate flavor-related expectations because they have strong associative ties with the sensory qualities of food and drink (see Cardello, 1994, 2007; Hutchings, 2003; Yeomans, Chambers, Blumenthal, \& Blake, 2008; Zellner \& Durlach, 2002; and Zellner, Strickhouser, \& Tornow, 2004, for a comprehensive review). These expectations likely result from our repeated exposure to the specific color-flavor pairings present in our environments (see Elliot \& Maier, 2007, for a review; cf. Garber, Hyatt, \& Boya, 2008; Garber, Hyatt, \& Starr, 2000, 2003; Tolman,
1938). According to Cardello (1994), a person's expectations about the sensory or hedonic properties of a food or drink can contribute just as much to his or her final assessments of that stimulus as the physiochemical properties of the food or drink itself (in the domain of food acceptance and hedonics; cf. Yeomans et al., 2008; see Feather, 1982; Masurovsky, 1939; Tolman, 1951).

Given the significant implications of this assertion, it is somewhat surprising that research investigating whether expectations play the same role in sensory evaluations (e.g., identification, intensity ratings, sweetness ratings, etc.) is in its infancy. Only a handful of studies in the literature to date have directly examined color's influence on orthonasal odor identification (Blackwell, 1995; Davis, 1981; Zellner et al., 1991; see Morrot, Brochet, \& Dubourdieu, 2001; Stevenson \& Oaten, 2008, for research that examines the influence of color on olfactory descriptions and discrimination). The earliest of these studies comes from Davis (1981). In Davis's experiment, the participants were instructed to smell odors that were applied, one per page, to the pages of a book. Participants were instructed to identify the odor present on the page and to write their response on the same page (they were also allowed to not respond). Twenty seconds later, they were instructed to turn the page and examine its contents, which contained either a color cue or nothing at all. Participants were then asked whether they would like to correct their responses (i.e., provide a second, different identification response) or instead give no response (i.e., stick with their initial response). Color cues were either color patches (e.g., a red color patch) or color words (e.g., the word red) that were deemed by the author to be either relevant (e.g., banana odor and the color yellow) or irrelevant (e.g., banana odor and the color red) to the odor in question. Davis reported that exposure to relevant cues evoked an increased rate of correct identification responses, which is to say that relevant color concepts facilitated participants' odor identification performance. By contrast, irrelevant cues reduced the rate of correct second responses.

Davis's (1981) interpretation of his experimental findings is somewhat problematic, since his study was quite prone to the effects of response bias (see Abdi, 2002). A more in-depth analysis of these results reveals that if no context cue was presented on the subsequent page, participants simply repeated their initial guess. By contrast, when a color cue of any kind (either relevant or irrelevant) was provided to participants, they showed an increased rate of second responses (although they did so to a greater degree for relevant color cues). When the color cue was relevant, a correct response was usually furnished, often following a nonanswer, and when the color cue was irrelevant, previously correct and nonresponses were converted into wrong answers. It is unclear from these results whether participants' responses reflected genuine colorodor interactions or whether, instead, they were simply reactions to what the participants believed the color cues were meant to suggest. This latter explanation seems especially likely, since participants were not allowed to smell the odor a second time once they were exposed to the color cue. Therefore, it seems probable that participants simply 
retroactively shifted their odor judgments into line with context cues that they believed were meant to help facilitate the correct identification of the odors.

Zellner et al. (1991) and Blackwell (1995), in an attempt to make color cues more contextually appropriate and less prone to task demands than in Davis's (1981) study, made the cues intrinsic to the drinks themselves. Participants in Zellner et al.'s study had to identify a number of odors under conditions in which the colors of the drinks themselves were appropriate, inappropriate, or else unavailable due to the blindfolding of the participant. (Appropriateness was determined on the basis of the results of a preliminary questionnaire, in which a different set of participants read a list of color names and wrote down the flavors that they would associate with each.) The authors reported that participants made fewer flavor identification errors when the drinks were appropriately colored than when they were either inappropriately colored or when participants had their eyes closed. ${ }^{1}$ In Blackwell's study, participants were presented with six different fruit drinks, four of which had incongruent color-flavor pairings and two of which had congruent pairings. They were asked to sniff each drink and to describe the odor. The authors reported that significantly more participants identified the odors correctly when the color of the fruit drink was appropriate (with respect to its odor identity) as compared with when it was inappropriate.

Although it is possible to explain the results of these three studies with an appeal to an "expectations"-based model - namely, that the visual cues in these experiments set up an expectation of the odor to be experienced as a consequence of prior learning-Cardello (1994) noted that in the sensory and food acceptance literature, "most studies have merely used the term [expectations] as a vague, post hoc explanatory variable. Expectations as a psychological construct have received no formal discussion and/or treatment in the sensory literature" (Cardello, 1994, p. 275). This same conclusion might be drawn for the three color-odor studies just discussed. Thus, the goal of the present research is to examine the cross-modal effect of color on odor identification by systematically examining how the flavor expectations generated by color cues might influence identification responses under a variety of different experimental conditions (see Wilson \& Klaaren, 1992, for a review of expectations-based models).

\section{An Individualized Approach to the Study of Expectancy Effects}

Before continuing, it is important to note that the present research makes one significant methodological departure from previous research on this topic; namely, it utilizes an "individualized" approach with regard to the assessment of participants' expectations about the flavors that are likely to be associated with specific colors. Cardello (2007) noted that individuals may have different expectations about what flavor a particular color indicates. Given that a person's "flavor history" (i.e., his or her exposure to specific copairings of flavors and colors) is undoubtedly idiosyncratic (see Shankar, Levitan, \& Spence, 2010), it seems imperative to control for any such differences when exploring expectations-based cross-modal effects on orthonasal odor identification.

However, the aforementioned studies (Blackwell, 1995; Davis, 1981; Zellner et al., 1991), in addition to other color-flavor studies, have largely overlooked such differences (see Davis, 1981; Dubose, Cardello, \& Maller, 1980; Zampini, Sanabria, Phillips, \& Spence, 2007; Zampini, Wantling, Phillips, \& Spence, 2008; Zellner et al., 1991). Interestingly, Cardello (2007) discussed the fact that, although most psychophysicists and sensory scientists have spent considerable time controlling for outside influences on their data, they have failed to control for more subtle, but potentially more significant (in terms of their consequences for response behaviors), influences that are brought to any experimental setting by the participant. Among these are physiological (e.g., taster status; see Zampini et al., 2008), cognitive (e.g., expectations), social, and cultural. Since all of these factors are much more difficult to control than, say, lighting in a testing booth, they have largely been overlooked in testing.

One example of an oversight in controlling for (potential) individual differences in color-flavor associations comes from Zellner et al. (1991). The authors determined which color-flavor combinations to use in their experiment on the basis of the results of a questionnaire in which a different group of participants responded with "the flavors they would expect a red, orange, yellow, green or purple drink to be."2 Therefore, the results generated in their experiment do not necessarily reflect violations or confirmations of expectations within specific individuals. The methodological improvement in the present experiments involved measuring the flavor expectations held by an individual and then examining whether or not the very same individual's flavor responses were influenced by his or her specific color-induced expectations. Furthermore, instead of using actual colors or colored drinks to measure people's expectations in preliminary testing, Zellner et al. (1991) used color words (e.g., a red drink or a purple drink). In the studies reported presently, the participants actually viewed specifically colored drinks, which controlled for any individual differences in what a color word such as red might inspire, in terms of hue, brightness, chroma, and so on. Finally, Zellner et al. (1991) analyzed all of their data in an aggregate, rather than in an individualized, fashion. This, of course, stemmed from the fact that the results of their preliminary testing were analyzed in an aggregate fashion. Cardello (1994, p. 254) pointed out (with regard to hedonic evaluations of foodstuffs) that

even if all consumers have the same perception of the intrinsic attributes of a product, the level of postinformation disconfirmation will differ from one consumer to the next [depending on his prior expectation] ... in such a situation, the difference in the mean expectation versus the mean baseline rating will reveal nothing about the levels and kinds of disconfirmation being experienced by the individual.

Thus, in the present experiments, we look at specific patterns of behavior within individuals first, depending on their responses to the particular color-flavor combinations 
that were tailored for them, before looking for more generalized patterns across the whole group of participants.

\section{An Expectations-Based Model for Understanding Color's Influence on Orthonasal Odor Identification}

The present research appeals directly to an affectbased model of expectations proposed by Wilson and Klaaren (1992). The hope is that insights from the affect expectations literature may provide a suitable framework for best conceptualizing the likely relationship between (color-based) expectations and more sensory-based evaluations, such as flavor identification.

Wilson and Klaaren (1992) stated that the relationship between people's expectations and the actual value of a stimulus can take on a number of forms. The first case represents a situation in which people have no prior expectations regarding a stimulus. In a case like this, evaluations of a stimulus are largely based on the objective and actual properties of the stimulus itself, since there is no expectation with which to compare it. The second case represents a situation in which the stimulus is entirely consistent with a person's expectation. Once a person has determined that his or her expectations have been met, his or her response is consistent with what would have been expected.

The third case, which will be the focus of the present experiments, is when the objective stimulus is discrepant from the expectation. When we interact with any stimulus, a comparative process takes place in which we directly compare and contrast the stimulus to any preexisting expectations we may have. At least two outcomes can result from this process. The first outcome is captured by assimilation theory. This model of behavior was initially proposed by Hovland, Harvey, and Sherif (1957) in order to help explain those behaviors in which people are willing to accept statements that differ from their own opinions and to adjust their attitudes on the basis of any prior expectations. According to this theory, when an expectation is unconfirmed (i.e., when stimulus attributes stand in contrast to what the individual's original expectation would have suggested), evaluations often shift toward one's expectations. Consequently, perceptions end up falling between the expected and actual stimulus.

In the domain of food and drink perception, an abundance of studies have shown an assimilation effect for both sensory and hedonic evaluations (Cardello \& Sawyer, 1992; Kahkonen \& Tuorila, 1998; Olson \& Dover, 1979; Schifferstein, Kole, \& Mojet, 1999; Tuorila, Cardello, \& Lesher, 1994; Wansink, van Ittersum, \& Painter, 2005; see Schifferstein, 2001, for a review). For example, in a study conducted by Olson and Dover (1979), expectations regarding the bitterness of a series of coffee beverages were generated in participants. The authors reported that participants who expected the coffee to taste bitter rated it as being significantly more bitter than when they evaluated the same coffee with little or no expectations of bitterness. Furthermore, Cardello and Sawyer reported that participants who expected to like a cola beverage rated this drink as more acceptable than when they evaluated the same drink with the expectations that they would like it less.
Similarly, Yeomans, Lartamo, Procter, Lee, and Gray (2001) found that giving tomato soup a name that connoted higher quality ("Gastronome's Connoisseur's Choice Cream of Tomato" as compared with "McTaggart's Lean and Low Tomato") led participants to rate the soup significantly more favorably (and as being significantly more creamy) than when no such label was given, even when the soups being evaluated had identical compositions. In other words, evaluations tended to shift into line with people's expectations. The results from these studies were interpreted to mean that the expected and actual experiences had combined in the final evaluation of the stimulus.

Contrast is another possible outcome. Contrast theory (see Schifferstein, 2001) refers to those conditions in which individuals engage in the aforementioned comparative process, but are unable to ignore or overlook any disparities between the expected and actual stimulus, in favor of shifting their evaluations into line with their expectations (see Yeomans et al., 2008; Zellner et al., 2004). As a result, they often focus more closely on the stimulus attributes themselves. In fact, it has been proposed that sometimes the surprise of the unexpected stimulus actually results in an exaggeration of the disparity between expected and actual stimulus properties. For example, in a study conducted by Yeomans et al. (2008), participants were presented with a novel food item, smoked-salmon mousse ice cream. When the food was labeled as "ice cream" (which generated strong expectations of a sweet fruit flavor), as compared with "frozen savory mousse" or "food 386," participants had a strong negative affective response to the food item and rated it as tasting more salty. In other words, contrast resulted when participants' expected (i.e., sweet and fruity) and actual (i.e., salty) sensory experiences were very different, which led to a decrease in the rated quality of the food and a higher salty rating than when the same food was evaluated without an expectation (see Anderson, 1973; Cardozo, 1965; Geers \& Lassiter, 1999; Marks \& Kamins, 1988, for other examples of contrast effects in the hedonics/consumer satisfaction literature).

Assimilation/contrast theory (see Zellner et al., 2004, for a review) combines these two theories and refers to the idea that both assimilation and contrast can occur as a result of a violation of prior expectation. In other words, participants may be willing to overlook stimulus attributes that differ from their expectations in certain conditions, but not in others. This effect was perhaps best captured by Zellner et al. (2004), who found that participants exhibited both assimilation and contrast even when identical stimuli were used in both conditions.

What determines whether assimilation or contrast occurs in any given context? In the present study, we examine how the degree of discrepancy between the expected stimulus and the actual stimulus (see Hovland et al., 1957; Wilson \& Klaaren, 1992; Zellner et al., 2004) might modulate the outcome of color's influence on participants' olfactory identification responses. Our prediction is that if the degree of perceptual discordance between the expected and actual flavor is small, these differences might be overlooked, and participants might be more inclined 
to shift their responses into line with their expectations (i.e., to assimilate; see Yeomans et al., 2008). On the other hand, if the discrepancy between the expected and actual stimulus is larger, the difference may be too great to still consider the expectation a reliable predictor of flavor identity (see Figure 1).

It is important that we make a few points regarding the application of assimilation/contrast theory to the present experiments. This theory has typically been used only to interpret responses that are more continuous in nature, such as the hedonic or sensory qualities of a food or drink item. In these contexts, it has been possible to properly quantify the extent to which responses lie between expected and actual experience (as in the case of assimilation), and the degree to which responses actually exaggerate noticed discrepancies (as in the case of contrast). However, since the present research examines response data that are categorical in nature (i.e., the identification of specific flavors), the extent to which (if at all) the content of one's expectation and experience is combined in the generation of the final flavor response cannot be measured reliably. This is because flavors do not have the same additive properties as numerical rat- ings. Similarly, although research in hedonics has looked at enhanced as compared with decreased evaluations, the categorical nature of flavors does not make this kind of analysis possible here. Thus, the definitions and use of the terms assimilation and contrast have been altered in order to account for the categorical nature of the present data. Assimilation will simply refer to a participant responding exactly in line with his or her flavor expectation (i.e., providing the same flavor response that he or she associated with a drink of that color in preliminary testing). Furthermore, since true contrast cannot be measured given the categorical nature of flavors, this term will not be used to represent a possible outcome of the present experiments. Instead, a participant will be said either to have assimilated or to have not assimilated (i.e., to have responded with a flavor that is not consistent with his or her expectation).

\section{The Present Experiments}

In running this series of experiments, our aim was first to determine whether color exerts an influence on the specific flavor identification responses generated under conditions of both low and high discrepancy, and then to

\section{Purple color generates expectation that drink will smell like "grape"}

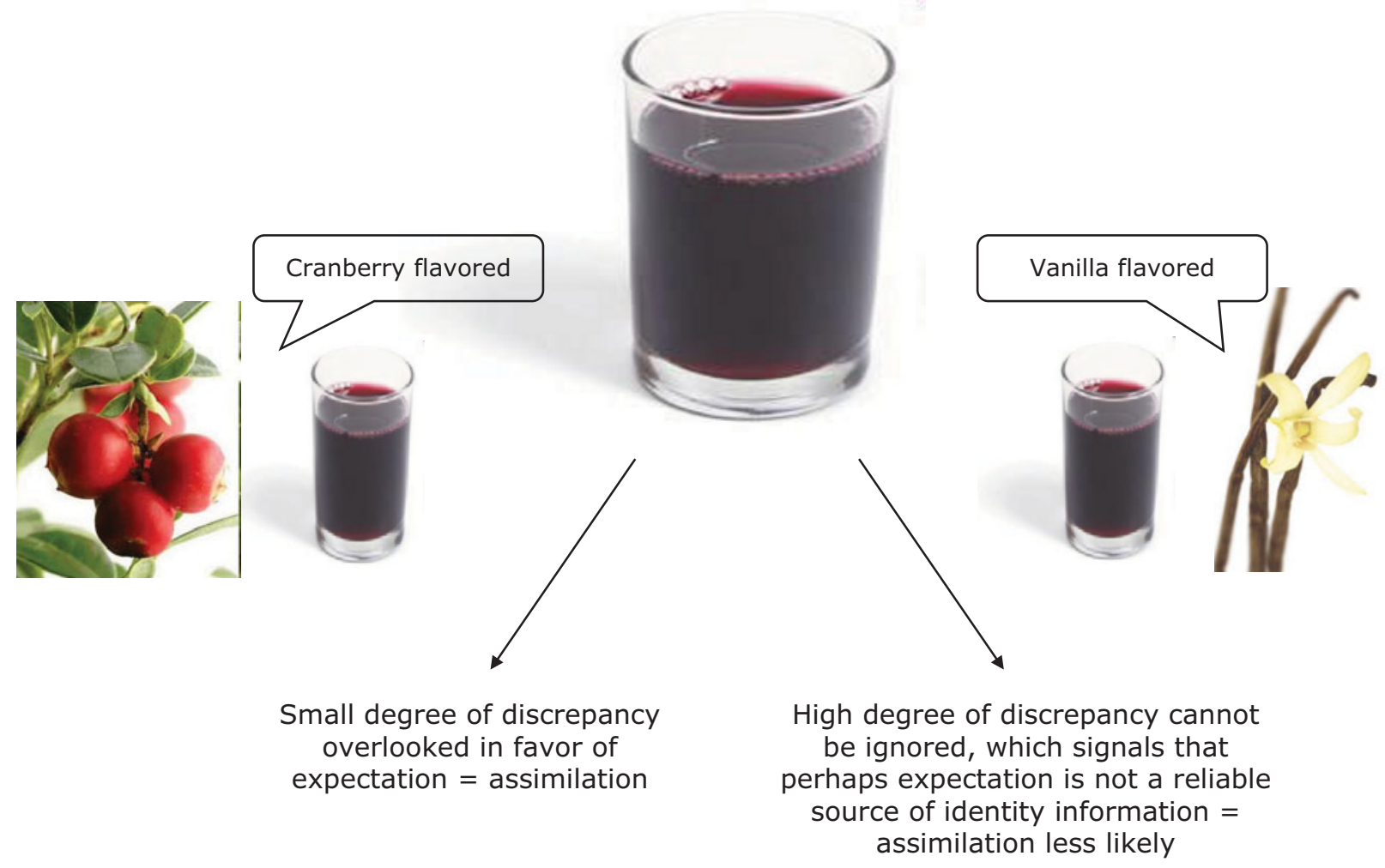

Figure 1. People typically see the color of a drink prior to smelling it (except in the case of drinking from a can). This color can set up a strong expectation as to what the drink's flavor will most likely be. This expectation can either be confirmed or disconfirmed through experience of the actual stimulus. The prediction is that if the degree of perceptual discordance between the expected and actual stimuli is small, differences may be overlooked in favor of a response that is consistent with this expectation. If, on the other hand, the discrepancy between the expected and actual stimuli is too large, differences may be too great to overlook and to still consider the expectation a reliable predictor of the drink's identity. As a result, assimilation may be less likely to occur. 
compare assimilation behavior between these two conditions. In preliminary testing, participants selected the one colored drink from among a lineup of drinks that inspired the strongest expectation of a particular flavor in their minds, and then they reported the flavor it made them think of. The chosen color will be referred to as a participant's "target color," and the chosen flavor will be referred to as a participant's "expected flavor." On the basis of this preliminary data, in Experiment 1, two flavored drinks were created for each individual that were of low discrepancy (LD) from each participant's expected flavor, ${ }^{3}$ and participants identified them by smell when the target color was added to them (color added condition) and when no color was added to them (no color added condition). Response behavior between these two conditions was then compared. In Experiment 4, the same colors were added to the drinks, but this time the flavors were of high discrepancy (HD) from each participant's expected flavor. The results from both of these experiments were then compared in order to see whether there was a significant difference in the effect of color on odor identification responses for low- and high-discrepancy stimuli.

\section{EXPERIMENT 1}

\section{Method}

Participants. Twenty North American (both by birth and nationality) participants were selected to participate in this experiment on the basis of their responses in a preliminary test ( 14 female, 6 male; normal or corrected-to-normal vision). In this test, participants viewed seven differently colored drinks and then chose the one drink from among the set that inspired the strongest expectation of a particular flavor, because it would "very unlikely be any other flavor but the one it first made them think of" (see Figure 2 for a photograph of the drinks presented and Table 1 for their color compositions). Participants then had to report the flavor that they thought a drink of that color would most likely be. Since the majority of participants chose either purple or orange as their target color (and chose grape and orange as their respective flavors), for the sake of experimental simplicity, only these participants were invited to participate in the experiment.

Apparatus, Stimuli, Design, and Procedure. Flavored drinks (each composed of just one flavor; see Table 2) were presented to participants with no color added to them (in order to get a baseline measure of the frequency with which participants reported the expected flavor for each drink in the absence of color cues) and also with the target color added to them. The frequency of expected fla-
Table 1

Drink Colors and Compositions

Colored Drink (Composition)

$\operatorname{Red}(0.16 \mathrm{~g} 10 \% \operatorname{Red} 40)$

Orange $(0.17 \mathrm{~g} 1.0 \%$ Yellow 6 \& $0.30 \mathrm{~g} .10 \%$ Red 40$)$

Yellow $(0.40 \mathrm{~g} .10 \%$ Yellow 5$)$

Green $(0.20 \mathrm{~g} .10 \%$ Yellow 5 \& $0.20 \mathrm{~g} \mathrm{.01 \%} \mathrm{Blue} 1)$

Blue $(0.27 \mathrm{~g} .10 \%$ Blue $1 \& 0.23 \mathrm{~g} .10 \%$ Red 40$)$

Purple $(0.45 \mathrm{~g} \mathrm{.10} \%$ Blue $1 \& 0.15 \mathrm{~g} 1.0 \%$ Red 40$)$ Pink (0.23 g .10\% Red 40)

Note-The table presents the compositions of the colored beverages that were presented to participants during preliminary testing. The drinks were made by adding $1 \mathrm{~g}$ of flavor with the required amount of food coloring and however much water was needed to yield a $100-\mathrm{g}$ solution. The same color compositions shown here were used in the creation of all experimental stimuli.

vor responses generated in the color-added condition was then compared with the frequency of such responses in the no-color-added condition. All of the trials were fully randomized within each block, and the order of the blocks was fully counterbalanced.

No color added. A total of eight drinks were made for each participant (see Table 2), two of which constituted critical trials (i.e., the two low discrepancy drinks), and the remainder of which constituted dummy trials. No color was added to any of these drinks. One gram of flavoring was added to $99 \mathrm{~g}$ of water in order to yield $100 \mathrm{~g}$ of drink, and drinks were presented to participants in 100-mL transparent plastic cups.

Color added. The same eight drinks as in the no-color-added condition were made, with the exception that this time they were colored (see Tables 1 and 2 for the drink types and their compositions).

In order to disguise the true nature of the experiment, participants were informed that they would be engaging in a "speeded identification task." (The experimenter pressed a stopwatch repeatedly throughout each experimental block in order to make it appear as though the participants' responses were being timed.) Participants were instructed to sniff each of the drinks, presented one at a time, and to report verbally the flavor identity of each drink to the experimenter. This response was then recorded on a computer by the experimenter. Participants' first responses were recorded unless they explicitly asked to change their initial response to another flavor.

Analysis. A test of independent proportions ${ }^{4}$ was used to compare participants' response behavior in the no-color-added and the coloradded conditions. Given the individualized nature of the data, it was important to code participants' responses according to a specific set of preordained rules: If participants responded with their expected flavor on a critical trial, this response was given a score of 1 . Otherwise, the response was given a score of 0 . The total scores from all
A

B

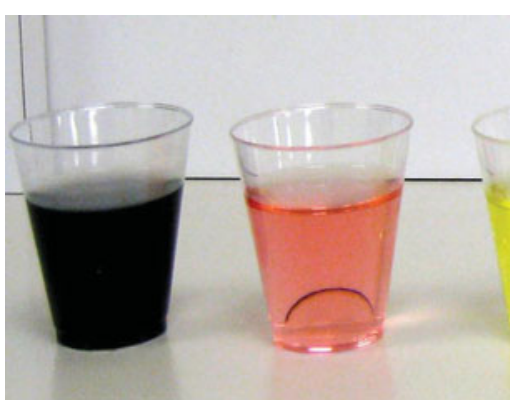

C
E

$\mathbf{F}$

G

Figure 2. The seven colored drinks shown to participants during preliminary testing. 
Table 2

Stimuli for Experiments 1-4

\begin{tabular}{cll}
\hline Target Flavor & \multicolumn{1}{c}{ Low-Discrepancy Experiment } & High-Discrepancy Experiment \\
\hline Grape & cranberry (purple), blueberry (purple) & banana (purple), vanilla (purple) \\
Orange & grapefruit (orange), lemon (orange) & watermelon (orange), mint (orange)
\end{tabular}

Dummy Trials raspberry (light blue), pineapple (yellow), strawberry (red), cherry (red), apple (green), lime (green)

Note-The table presents the test stimuli (and their respective colors for use in the color added condition; see Table 1 for compositions) created for both Experiment 1 (low discrepancy) and Experiment 2 (high discrepancy). Dummy trial drinks and their respective colors are listed in the final row of the table.

responses were tallied for each condition, and the two proportions generated from both the color-added and no-color-added conditions were then compared.

In order to tease out the specific influence of color on the generation of expected flavor responses from other possible influences (such as the influence of the perceptual similarity between the expected and actual flavors - e.g., cranberry and grape may simply be confusable flavors), the score associated with each person's response for a particular flavored drink in the no-color-added condition was subtracted from the score assigned to his or her response for that same flavored drink in the color-added condition. Resulting scores of " 1 " indicated cases in which responses assimilated, as compared with scores of "0" (note that scores of "-1" were similarly coded as "0," since responding with the expected flavor in the no-color-added condition but not in the color-added condition did not reflect a case of assimilation).

\section{Results}

In the no-color-added condition, $10 \%$ of flavor responses (i.e., 4/40 total responses) were consistent with each participant's expected flavor (i.e., either grape or orange, depending on each person's preliminary results). By contrast, in the color-added condition, $40 \%$ of responses (16/40 total responses) were consistent with each participant's expected flavor. A test of independent proportions revealed a significant difference in the proportion of "expected flavor" responses generated between these two conditions $(p<.001$; see Figure 3$)$. In other words, there was a significantly greater proportion of grape or orange responses when the critical drinks were colored in a manner that was congruent with participants' expectations (i.e., purple and orange) as compared with when they were not. These results indicate that color has a significant effect on participants' response behavior when the degree of discrepancy between the expected and actual flavors is small. Thirty-three percent of responses (13/40 total responses) shifted into line with participants' expectations (i.e., assimilated) due to the influence of the target color.

In Experiment 2, color's influence on participants' flavor identification responses was examined when they were explicitly told that the color cues might be irrelevant and should thus be ignored. Of interest was the degree to which the effect of color on flavor identification seen in Experiment 1 depends on cognitive/response biases, such as the belief that color is meant to be an informative cue.

\section{EXPERIMENT 2}

\section{Method}

Participants. Eighteen North American participants (11 female, 7 male; normal or corrected-to-normal vision) were selected to take part in this experiment at Givaudan on the basis on their results in the same preliminary testing task reported in Experiment 1. Participants had had prior exposure to these same stimuli in weeks prior. They were paid in return for their participation.

Apparatus, Stimuli, Design, and Procedure. Experiment 2 was identical to Experiment 1, with the exception that the instruc-

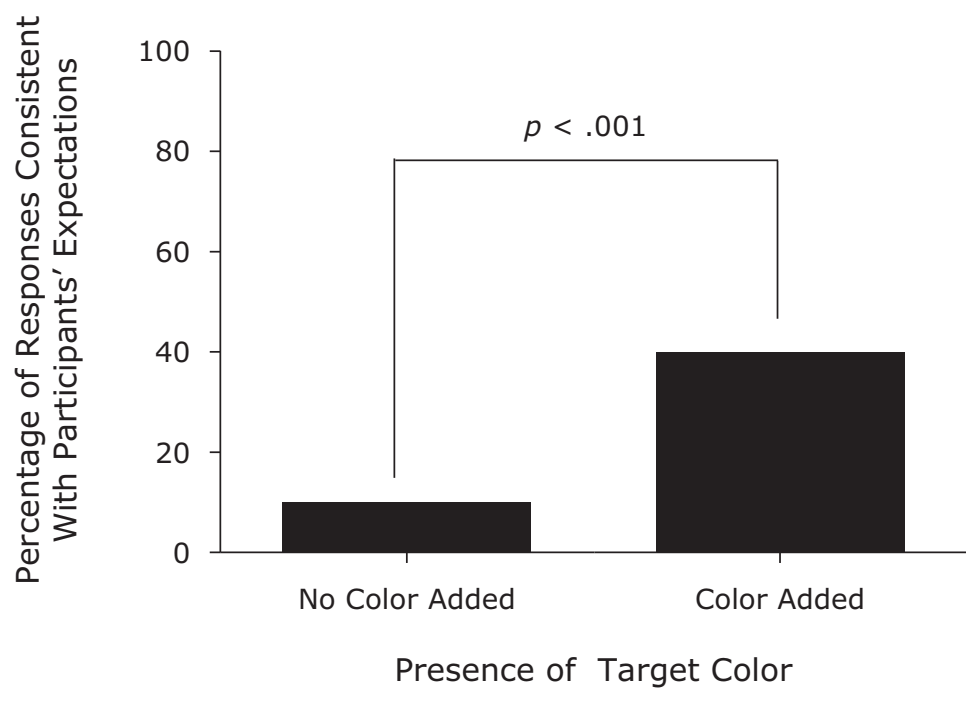

Figure 3. The influence of the target color on the percentage of responses that were consistent with participants' expected flavor in Experiment 1. 
tions in the color-added condition were altered slightly. The experimenter informed the participants that the colors of the drinks would be uninformative cues and should thus be ignored.

\section{Results and Discussion}

Six percent of flavor responses (2/36 total responses) were consistent with each participant's expected flavor in the no-color-added condition. When explicit instructions were given to participants that the color of the drinks would be irrelevant with respect to flavor identity, $28 \%(10 / 36)$ of responses were consistent with each participant's expected flavor. A test of independent proportions once again revealed a significant difference between the proportion of expected flavor responses generated between these two conditions ( $p=.006$; see Figure 4$)$. Twenty-five percent of responses (9/36 total responses) assimilated to participants' expectations. Interestingly, no significant difference was found between the results of this experiment and Experiment 1 , according to the results of a test of independent proportions ( $p=.236$; see Figure 4$)$. These results reveal that even when participants are informed that color cues will not be informative, color still has a significant influence on their identification responses, suggesting, perhaps, that the effect of color on flavor identification is automatic, so long as the degree of discrepancy between the expected and actual flavors is small.

The goal of Experiment 3 was to examine whether lessening the response demands associated with this task (in addition to instructions indicating the irrelevance of color) might make participants more certain of flavor identity and thus lessen the potential influence of color on their identification responses. A near identical version of Experiment 2 was conducted, except that this time the participants were told that they would be engaging in an accuracy task. Participants were instructed to take as much time as they needed when making their judgments.

\section{EXPERIMENT 3}

\section{Method}

Participants. Fifteen North American participants (10 women, $5 \mathrm{men}$; normal or corrected-to-normal vision) were selected to participate in this experiment at Givaudan on the basis of their results in the same preliminary testing task reported in Experiment 1. Participants were paid in return for their participation.

Apparatus, Stimuli, Design, and Procedure. Experiment 3 was identical to Experiment 2, with the additional instructions that participants would be taking part in an accuracy task and should take as much time as they needed before making their responses.

\section{Results and Discussion}

In the no-color-added condition, $7 \%$ of flavor responses (i.e., 2/30 total responses) were consistent with each participant's expected flavor (i.e., either grape or orange, depending on each person's preliminary results). By contrast, in the color-added condition, $23 \%$ of responses $(7 / 30$ total responses) were consistent with each participant's expected flavor. A test of independent proportions revealed a significant difference between the proportion of expected flavor responses generated in these two conditions ( $p=$ .035; see Figure 4).

Seventeen percent of responses (5/30 total responses) assimilated to participants' expectations. No significant difference was found between response behavior in this experiment and that in Experiment 2, according to the results of a test of independent proportions ( $p=.205$; see Figure 4). Thus, even when participants are not placed under time pressure, their judgments are nevertheless significantly influenced by the color cues present in the drinks that they evaluate. This suggests once again that the effect of color on flavor identification may be automatic and not wholly dependent on cognitive/task demands. In Experiment 4, we examined the influence of color on flavor identification under conditions of high discrepancy.

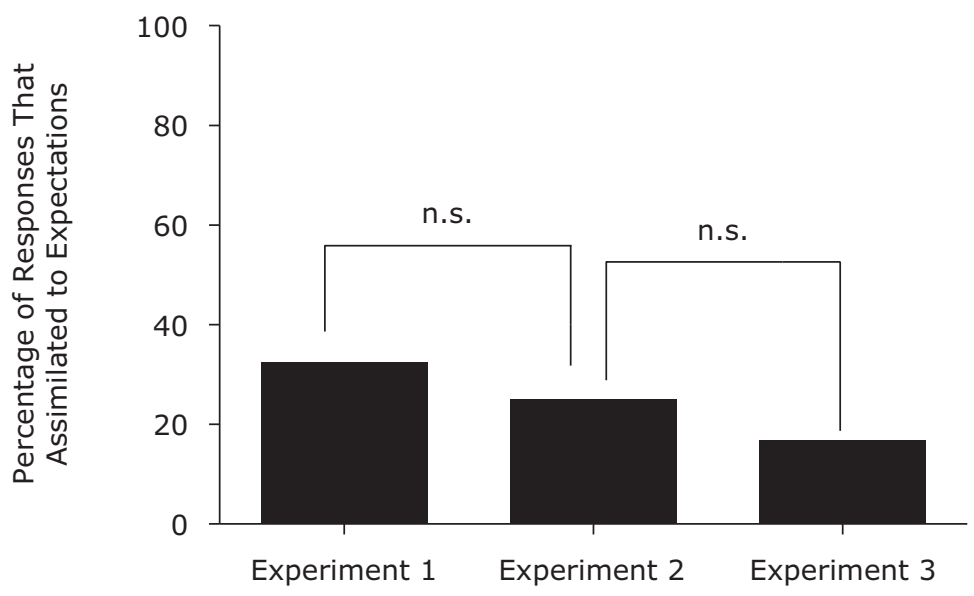

Figure 4. The influence of the target color on the percentage of responses that assimilated to participants' expectations in Experiment $1(N=20)$, Experiment 2 (when they were told that color would be an uninformative cue; $N=18$ ), and Experiment 3 (when they were told that color would be an uninformative cue and were given as much time as they desired to complete the task; $N=15$ ). All of the manipulations resulted in a significant effect of color on the proportion of responses that were consistent with participants' expectations. 


\section{EXPERIMENT 4}

\section{Method}

Participants. The same participants who took part in Experiment 1 took part in Experiment 4.

Apparatus, Stimuli, Design, and Procedure. These were identical to those in Experiment 1, with the exception that the two critical drinks were of high discrepancy from each participant's expected flavor.

\section{Results and Discussion}

In the no-color-added condition, $0 \%$ of flavor responses (i.e., 0/40) were consistent with each participant's expected flavor (i.e., either grape or orange, depending on each person's preliminary results). Similarly, in the coloradded condition, $0 \%$ of responses $(0 / 40)$ were consistent with each participant's expected flavor. Thus, there was no significant difference in response behavior between these two conditions (see Figure 5). These results indicate that color does not have a significant effect on the proportion of responses that are consistent with participants' expectations when the degree of discrepancy between the expected and actual flavors is larger. Zero percent of responses ( $0 / 40$ total responses) assimilated to participants' expectations because of the influence of the target color.

There was a significant difference $(p<.001$; see Figure 6) in the proportion of responses that assimilated to participants' expectations in Experiment 2 (low discrepancy) as compared with Experiment 4 (high discrepancy).

The prediction here had been that if the degree of discrepancy between the actual and expected flavors was large, the presence of a color strongly associated with a particular flavor would have little effect in terms of shifting participants' responses toward this expectation. Indeed, no significant effect of color on the proportion of responses that were consistent with participants' expectations in the color added as compared with the no color condition was found in Experiment 5. Critically, there was a significant difference in assimilation behavior between the low and high discrepancy Experiments (Experiments 1 and 4, respectively). A significantly greater proportion of responses assimilated toward participants' expected flavor in Experiment 1 as compared with that in Experiment 4, suggesting that the degree of discrepancy between the actual and expected flavors is able to mediate color's influence on participants' flavor identification responses. This result is particularly compelling, given that the same target colors were used in both experiments. Therefore, differences in assimilation between the two experiments reported in the present article cannot be reduced to differences in any given color's susceptibility to multisensory influence. Furthermore, the fact that the same colors were used in both conditions provides perhaps the strongest argument against the idea that it was simply response bias that drove the results. If participants believed that they ought to respond in line with the color because it would yield the right answer or appease the experimenter, an effect of color should have been noted in both experiments. This was clearly not the case.

\section{GENERAL DISCUSSION}

The goal of the present experiments was to examine the degree of discrepancy between the expected flavor of a drink and the actual flavor of a drink, in order to see whether it would modulate the cross-modal influence of color on flavor identification (as based on orthonasal olfaction). The prediction was that if the degree of perceptual discordance between the expected and actual flavor was small, color would likely exert a significant influence on the proportion of responses that were consistent with participants' expected flavor. On the other hand, if the discrepancy between the two was larger, the prediction was

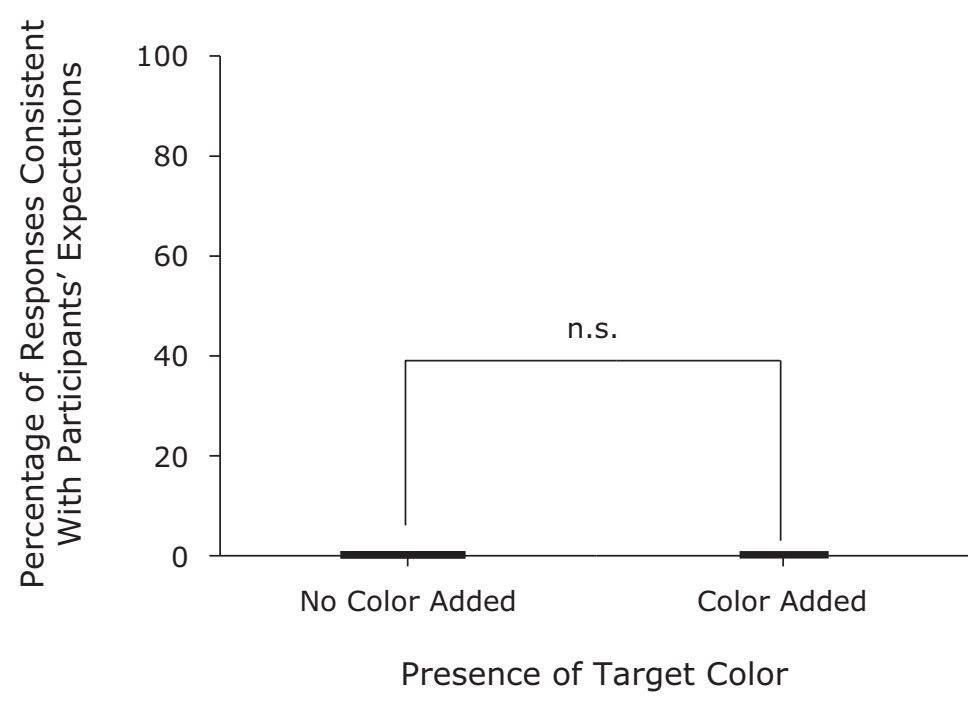

Figure 5. The influence of the target color on the percentage of responses that were consistent with participants' expected flavor in Experiment 4. 


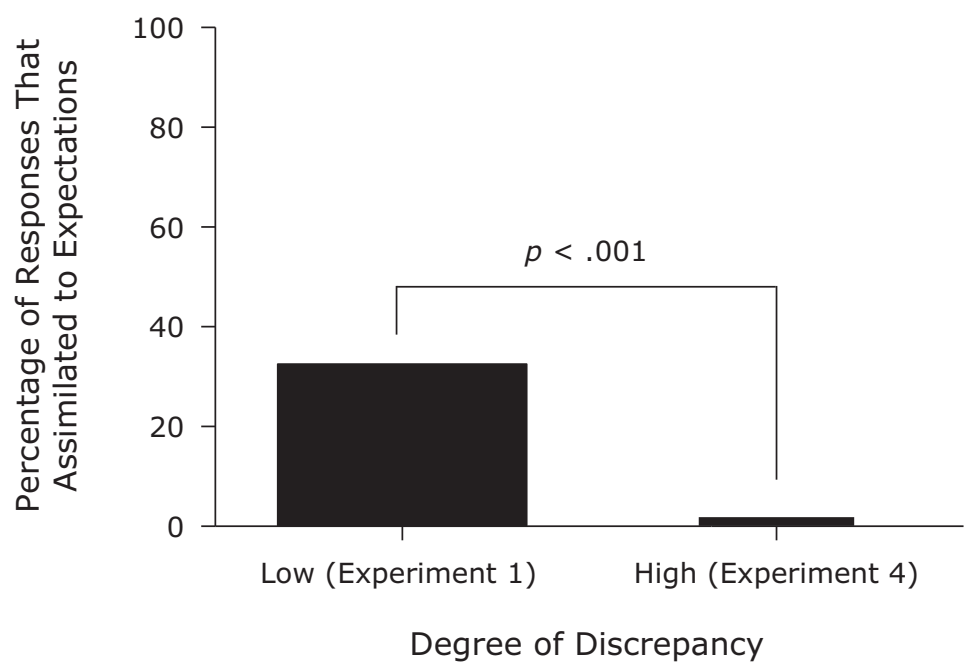

Figure 6. The influence of the target color on the percentage of responses that assimilated to participants' expectations as a function of the degree of discrepancy.

that the visually induced expectation would no longer be seen as a reliable cross-modal predictor of a drink's flavor identity. Thus, participants ought to be less influenced by their color-generated expectation and less inclined to respond with their expected flavor.

These predictions were supported by the results of the present experiments. In the low-discrepancy experiment (Experiment 1), a significant difference was found between the proportion of responses that were consistent with participants' expected flavor in the color-added as compared with the no-color-added conditions (see Figure 3). This result did not simply reflect some kind of cognitive demand characteristic, according to the results of Experiments 2 and 3 (see Figure 4).

In the high-discrepancy experiment (Experiment 4), no significant difference was reported between the proportion of responses that were consistent with participants' expected flavor in the color-added as compared with the no-color-added conditions. Thus, when the degree of discrepancy between participants' expected flavor and the actual flavor was larger (relative to the degree of discrepancy in Experiment 1), color cues did not shift responses into line with this expectation (see Figure 5). Perhaps most importantly, a significant difference in assimilation behavior was observed between Experiments 1 and 4.

\section{Theoretical Accounts That May Help to Explain These Results}

Assimilation/contrast theory refers to the idea that participants may be willing to overlook stimulus attributes that differ from their expectations in certain conditions but not in others. This theory is consistent with findings from other senses that have looked at interactions between incongruent pairs of stimuli presented to different modalities. For instance, the effect of auditory-visual discrepancies has often been studied. In one of the earliest examples, Jackson (1953) conducted an experiment in which he presented auditory and visual stimuli at different locations and asked his participants to indicate the source of the sound. When discrepancies were small, participants were biased by the location of the visual stimulus, but when discrepancies grew larger, they essentially discounted the information before their eyes. The assimilation contrast model, coupled with the Bayesian decision model (see Ernst, 2005) and the information reliability hypothesis (see Andersen, Tiipana, \& Sams, 2005), are proposed as the most tenable means of explaining the modulating role of color-based expectancy information on participants' identification responses.

According to Schifferstein (2001),

the most important aspect of Assimilation-Contrast Theory is that the size of the discrepancy between expected and actual product performance determines the way subjects deal with the disparity. ... A final product evaluation is determined by the discrepancy between the product's properties as perceived during trial and the a priori expectations for those properties. (pp. 78, 87)

In other words, when discrepancies are small, individuals tend to overlook them, because the stimulus still falls within a reasonable latitude of acceptance based on their expectations. In an attempt to reduce the small cognitive dissonance that exists, individuals will minimize any perceived discrepancy and assimilate their responses to their expectations. On the other hand, when discrepancies are larger, the stimulus may now fall within a latitude of rejection. Thus, the individual's expectation may no longer be seen as a reliable reference, and assimilation does not occur.

The results reported in the present article seem to be consistent with this model (see Schifferstein, 2001, for a review of assimilation/contrast theory). We often rely on higher level cognition and other sensory cues, such as color, to help us identify odors and their associated objects. Thus, 
our judgments of the identities of various drinks are subject to cross-modal influences. However, it seems reasonable to assume that the expectations generated by color will be used as a reliable predictor of odor only if they fall within a reasonable latitude of acceptance (see Yeomans et al., 2008). Presumably, in Experiment 1, people's expectations were not so strongly violated that they no longer saw color cues as a reliable source of flavor identity information. However, in Experiment 4, the contrast between the expected flavor (derived from color) and the actual flavor (derived from orthonasal olfactory cues) was so discrepant, the content of people's expectations was most likely disregarded in the final assessment of flavor identity.

One of the underlying assumptions of the assimilation/ contrast model is that preexisting knowledge structures have the ability to influence our evaluative judgments. In Experiments 1-3, expectancy information-that is, the flavor expectations generated by color cues - was, in fact, able to influence participants' orthonasal odor identification responses, just so long as the degree of discrepancy between participants' expected flavor and the actual flavor of the drinks was small. The importance of top-down information in mediating such cross-modal effects has been acknowledged by a number of researchers who have explored expectancy effects using this model in other domains of psychology. For example, with regard to the perception of food products, Schifferstein (2001, p. 73) noted that

when consumers taste a food product in a real-life situation, their perception of the product is not only based on the sensory characteristics of the product per se. Product perception is often biased by preconceived ideas about product properties and is affected by the consumer's judgmental frame of reference.

Similarly, Wilson and Klaaren (1992) discussed the fact that most theories of perception, memory, judgment, person perception, and self-perception are increasingly moving in the direction of top-down models. They are being constructed to account for the strong role that knowledge structures (e.g., schemata, theories, categories, models, expectancies, hypotheses, etc.) have been shown to play in our sensory judgments.

In fact, the important role of such higher level cognitive factors in mediating cross-modal interactions is inherent to one of the most prominent theories in the field of multisensory perception-namely, the Bayesian decision model (see Ernst, 2005, for a review). Ernst and Bülthoff (2004, p. 164) stated, with regard to the problem of the perception, that

a reconstruction of the environment based on sensory data has to be formed in the brain. It is however impossible to reconstruct the environment "bottom-up" from the sensory information alone. Prior knowledge is needed to interpret ambiguous sensory information. Bayesian inference provides a formal way to describe such interactions and enables one to model the uncertainty about the world by combining prior knowledge (that might be unconscious) with ob- servational, sensory evidence (the likelihood function) to infer the most probable interpretation of the environment.

Thus, researchers in the field of multisensory perception have increasingly come to the view that stimulus information is combined with a priori knowledge/expectations in order to form our posterior representation of those objects and events in the world around us. Given that orthonasal olfactory cues on their own have been shown to be insufficient with regard to correct odor identification, it is to be expected that participants may look to other sources of information (i.e., color-based expectations) to facilitate correct odor identification. The present data are consistent with another feature of Bayesian decision theory, which asserts that maximizing information delivered from the different senses is a critical strategy for processing different sensory signals, in order to maximally reduce the variance associated with the final multisensory estimates (Ernst \& Bülthoff, 2004).

The importance of these factors in mediating such crossmodal effects parallels the theoretical framework of the information reliability hypothesis, which has previously been presented to explain audiovisual fusion in speech perception (Andersen et al., 2005; Schwartz, Robert-Ribes, \& Escudier, 1998; Wada, Kitagawa, \& Noguchi, 2003). The hypothesis here is that the modality providing the most reliable information dominates. In the context of the present experiments, reliability refers to the modality thought to provide the most reliable flavor-based information.

To the best of our knowledge, this is the first research to reveal that a variable such as the degree of discrepancy can significantly influence the extent to which participants' flavor-based expectations are used in orthonasal olfactory judgments. Future research should examine other variables that can mediate whether a person's expectations influence his or her final judgments of a stimulus. The strength of a person's expectation, his or her level of expertise, the spatial and temporal presentation of color cues relative to the presentation of odor cues, age, and taster status (see Shankar, Simons, Shiv, Levitan, et al., in press; Shankar, Simons, Shiv, McClure, \& Spence, in press; Zampini et al., 2008) all appear to be relevant variables that may affect the degree to which color cues influence odor identification.

Perhaps more importantly, the present research introduces a new methodology for examining color-flavor interactions. We measured the flavor expectations held by an individual and then examined whether the very same individual was influenced by his or her specific expectations before looking for more general trends across the group as a whole. By designing stimuli that were specifically tailored to each individual's expectations, we were able to investigate whether those expectations could predict the pattern of odor misidentification that results when a target color is added to a food or drink. We believe that if the goal is to create a comprehensive and predictive model of cross-modal interactions in future research, it is imperative to control for an individual's expectations and to examine those factors that can interact with these expectations. 


\section{AUTHOR NOTE}

Correspondence concerning this article should be addressed to M. Shankar, Department of Psychology, 420 Jordan Hall, Stanford University, 450 Serra Mall, Stanford, CA 94305 (e-mail: mayas@stanford.edu).

\section{REFERENCES}

ABDI, H. (2002). What can cognitive psychology and sensory evaluation learn from each other? Food Quality \& Preference, 13, 445-451.

Andersen, T., Tirppana, K., \& Sams, M. (2005). Maximum likelihood integration of rapid flashes and beeps. Neuroscience Letters, 380, 155 160 .

ANDERSON, R. (1973). Consumer dissatisfaction: The effect of disconfirmed expectancy on perceived product performance. Journal of Marketing Research, 10, 38-44.

Auvray, M., \& Spence, C. (2008). The multisensory perception of flavor. Consciousness \& Cognition, 17, 1016-1031.

BlackWell, L. (1995). Visual cues and their effects on odor assessment. Nutrition \& Food Science, 5, 24-28.

CAIN, W. (1979). To know with the nose: Keys to odor identification. Science, 203, 467-470.

Candolin, U. (2003). The use of multiple cues in mate choice. Biological Review, 78, 575-595.

CARdello, A. (1994). Consumer expectations and their role in food acceptance. In H. J. H. MacFie \& D. M. H. Thomson (Eds.), Measurement of food preferences (pp. 253-297). London: Blackie.

CARdello, A. (2007). Measuring consumer expectations to improve food product development. In H. J. H. MacFie (Ed.), Consumer-led food product development (pp. 223-261). Cambridge: Woodhead.

CARDELlO, A., \& SAWYER, F. (1992). Effects of disconfirmed consumer expectations of food acceptability. Journal of Sensory Studies, 7, 253277.

CARdozo, R. (1965). An experimental study of customer effort, expectation, and satisfaction. Journal of Marketing Research, 2, 244-249.

DAVIS, R. (1981). The role of nonolfactory context cues in odor identification. Perception \& Psychophysics, 30, 83-89.

Desor, J. A., \& Beauchamp, G. K. (1974). The human capacity to transmit olfactory information. Perception \& Psychophysics, 16, 551-556.

DuBose, C. N., Cardello, A. V., \& Maller, O. (1980). Effects of colorants and flavorants on identification, perceived flavor intensity, and hedonic quality of fruit-flavored beverages and cake. Journal of Food Science, 45, 1393-1399.

Elliot, A., \& Maier, M. (2007). Color and psychological functioning. Current Directions in Psychological Science, 16, 250-254.

Engen, T., \& Ross, B. (1973). Long-term memory of odors with and without verbal descriptions. Journal of Experimental Psychology, 100, 221-227.

ERNST, M. (2005). A Bayesian view on multimodal cue integration. In G. Knoblich, M. Grosjean, I. Thornton, \& M. Shiffrar (Eds.), A Bayesian view on multimodal cue integration (pp. 105-131). New York: Oxford University Press.

ERnst, M., \& BüLthoff, H. (2004). Merging the sense into a robust percept. Trends in Cognitive Sciences, 8, 162-169.

FEATHER, N. (1982). Expectations and actions: Expectancy-value models in psychology. Hillsdale, NJ: Erlbaum.

Garber, L., Hyatt, E., \& Boya, Ü. (2008). The mediating effects of the appearance of nondurable consumer goods and their packaging on consumer behavior. In H. N. J. Schifferstein \& P. Hekkert (Eds.), Product experience (pp. 581-602). Amsterdam: Elsevier.

Garber, L., Hyatt, E., \& Starr, R. (2000). The effect of food color on perceived flavor. Journal of Market Theory Practice, 8, 59-72.

Garber, L., Hyatt, E., \& Starr, R. (2003). Measuring consumer response to food products. Food Quality \& Preference, 14, 3-15.

GeERs, A., \& Lassiter, G. (1999). Affective expectations and information gain: Evidence for assimilation and contrast effects in affective experience. Journal of Experimental Social Psychology, 35, 394-413.

Herz, R., \& von Clef, J. (2001). The influence of verbal labeling on the perception of odors: Evidence for olfactory illusions? Perception, 30, 381-391.

Hovland, C., Harvey, O., \& Sherif, M. (1957). Assimilation and contrast effects in reactions to communication and attitude change. Journal of Abnormal \& Social Psychology, 55, 244-252.
Hutchings, J. (2003). Expectations and the food industry: The impact of color and appearance. New York: Kluwer Academic/Plenum.

JACKSON, C. V. (1953). Visual factors in auditory localization. Quarterly Journal of Experimental Psychology, 5, 52-65.

KAHKONEN, P., \& TUORILA, H. (1998). Effect of reduced-fat information on expected and actual hedonic and sensory ratings of sausage. Appetite, 30, 13-23.

MarKs, L., \& Kamins, M. (1988). The use of product sampling and advertising: Effects of sequence of exposure and degree of advertising claim exaggeration on consumers' belief strength, belief confidence, and attitudes. Journal of Marketing Research, 25, 266-281.

Masurovsky, B. (1939). How to obtain the right food color. Food Engineering, 11, 55-59.

Morrot, G., Brochet, F., \& Dubourdieu, D. (2001). The color of odors. Brain \& Language, 79, 309-320.

OlSON, J., \& Dover, P. (1976). Effects of expectation creation and disconfirmation on belief elements of cognitive structure. In D. D. Anderson (Ed.), Advances in consumer research (Vol. 3, pp. 168-175). Provo, UT: Association for Consumer Research.

OLSON, J., \& Dover, P. (1979). Disconfirmation of consumer expectations through product trial. Journal of Applied Psychology, 64, 179-189.

RABIN, M., \& CAIN, W. (1984). Odor recognition: Familiarity, identifiability, and encoding consistency. Journal of Experimental Psychology: Learning, Memory, \& Cognition, 10, 316-325.

RichaRdSON, J., \& ZuCCO, G. (1989). Cognition and olfaction: A review. Psychological Bulletin, 105, 352-360.

Sснав, F. (1991). Odor memory: Taking stock. Psychological Bulletin, 109, 242-251.

SCHIFFERSTEIN, H. (2001). Effects of product beliefs on product perception and liking. In L. Frewer, E. Risvik, \& H. Schifferstein (Eds.), Food, people and society: A European perspective of consumers 'food choices (pp. 73-96). London: Springer.

Schifferstein, H., Kole, A., \& Mojet, J. (1999). Asymmetry in the disconfirmation of expectations for natural yogurt. Appetite, 32, 307-329.

SchwartZ, J., Robert-Ribes, J., \& Escudier, P. (1998). Ten years after Summerfield: A taxonomy of models for audiovisual fusion in speech perception. In R. Campbell, B. Dodd, \& D. Burnham (Eds.), Hearing by eye, II: Perspectives and directions in research on audiovisual aspects of language processing (pp. 85-108). Hove, U.K.: Psychology Press.

Shankar, M., Levitan, C., \& Spence, C. (2010). Grape expectations: The influence of cognitive influences in color-flavor interactions. Consciousness \& Cognition, 19, 380-390.

Shankar, M., Simons, C., Shiv, B., Levitan, C., McClure, S., \& SPENCE, C. (in press). An expectations-based approach to explaining the cross-modal influence of color on orthonasal odor identification: The influence of temporal and spatial factors. Journal of Sensory Studies.

Shankar, M., Simons, C., Shiv, B., McClure, S., \& Spence, C. (in press). An expectations-based approach to explaining the cross-modal influence of color on orthonasal odor identification: The influence of expertise. Chemosensory Perception.

STEVEnson, R. (2009). The psychology of flavour. Oxford: Oxford University Press.

Stevenson, R., \& OAten, M. (2008). The effect of appropriate and inappropriate stimulus color on odor discrimination. Perception \& Psychophysics, 70, 640-646.

Tolman, E. (1938). The determiners of behaviors at a choice point. Psychological Review, 45, 1-45.

Tolman, E. (1951). Behavior and psychological man. Berkeley: University of California Press.

Tuorila, H., Cardello, A., \& Lesher, L. (1994). Antecedents and consequences of expectations related to fat-free and regular-fat foods. Appetite, 23, 247-263.

Verhagen, J. V., \& Engelen, L. (2006). The neurocognitive bases of human multimodal sensory integration. Neuroscience \& Biobehavioral Reviews, 30, 613-650.

Vlahos, J. (2006). The smell of war. Popular Science, 8, 72-95.

Wada, Y., Kitagawa, N., \& Noguchi, K. (2003). Audio-visual integration in temporal perception. International Journal of Psychophysiology, 50, 117-124.

WANSINK, B., VAN ItTERSUM, K., \& PAINTER, J. (2005). How descriptive food names bias sensory perceptions in restaurants. Food Quality \& Preference, 16, 393-400. 
Wilson, T. D., \& KLAAREN, K. (1992). The role of affective expectations in affective experiences. In M. S. Clear (Ed.), Review of personality and social psychology: Emotion and social behavior (pp. 1-31). Newbury Park, CA: Sage.

Yeomans, M., Chambers, L., Blumenthal, H., \& Blake, A. (2008). The role of expectancy in sensory and hedonic evaluation: The case of smoked salmon ice-cream. Food Quality \& Preference, 19, 565-573.

Yeomans, M., Lartamo, S., Procter, E., Lee, M., \& Gray, R. (2001). The actual, but not labeled, fat content of a soup alters short-term appetite in healthy men. Physiology \& Behavior, 73, 533-540.

Zampini, M., Sanabria, D., Phillips, N., \& Spence, C. (2007). The multisensory perception of flavor: Assessing the influence of color cues on flavor discrimination responses. Food Quality \& Preference, 18, 975-984.

Zampini, M., Wantling, E., Phillips, N., \& Spence, C. (2008). Multisensory flavor perception: Assessing the influence of fruit acids and color cues on the perception of fruit-flavored beverages. Food Quality \& Preference, 19, 335-343.

Zellner, D., Bartoli, A., \& Eckard, R. (1991). Influence of color on odor identification and liking ratings. American Journal of Psychology, 104, 547-561.

Zellner, D., \& Durlach, P. (2002). Effect of color on expected and experienced refreshment, intensity, and liking of beverages. American Journal of Psychology, 116, 633-647.

Zellner, D., Strickhouser, D., \& Tornow, C. (2004). Disconfirmed hedonic expectations produce perceptual contrast, not assimilation. American Journal of Psychology, 117, 363-388.

\section{NOTES}

1. It should be noted that in Zellner et al.'s (1991) odor identification experiments, the word flavor (as compared with odor) was used in the instructions to participants. Presumably, this is because the word flavor is a more colloquial and well-understood concept among the general population and often refers to the odorant that is added to any drink. In all of the presently reported experiments, participants were also asked to provide responses regarding their "flavor" expectations and judgments for the presented drinks. Similarly, just as in Zellner et al.'s articles, the term flavor in this manuscript will be used to refer to the olfactory component of each drink stimulus. This use of this word should not be confused with flavor perception, which refers to a multisensory process involving multiple senses (see Stevenson, 2009, for a review).

2. Note that Davis (1981) and Blackwell (1995) did not even collect this kind of data. Instead, they used their personal judgments to determine which color-flavor combinations were to be considered as either "appropriate" or "inappropriate."

3. Because decisions regarding what constituted a "low" discrepancy (LD) or a "high" discrepancy (HD) drink were decided by a senior application scientist at Givaudan, a control condition was conducted in order to determine whether the selected drinks were in fact perceived as such by participants. Ten drinks (that did not have any color added to them) were made for this condition and were divided into two blocks: purple and orange. For the purple block (Block 1), five drinks were made: grape (reference), cranberry (LD), blueberry (LD), banana (HD), and vanilla (HD). For the orange block, five drinks were made: orange (reference), grapefruit (LD), lemon (LD), watermelon (HD), and mint (HD). All of the drinks were made by adding $1 \mathrm{~g}$ of flavorant to $99 \mathrm{~g}$ of water. A different group of participants $(N=20)$ participated in the block that matched their results from preliminary testing. The drinks were presented to each participant on a white table in front of them. They were instructed to sniff the reference drink and to rank the remaining four drinks in order from most similar to least similar to the reference. A Friedman test statistic revealed that the ranking orders for the low discrepancy drinks were significantly different from those of the high discrepancy drinks, with the LD drinks rated as more similar to the reference than were the HD drinks $(p<.05)$.

4. A test of independent proportions was used because the structure of the data did not meet the requirements of a test of dependent proportions. However, since independent-sample tests are more conservative than dependent samples tests, the significant results that were obtained are still valid. Note that if a dependent test had existed, it would have likely found these same differences, only they would have been at a higher significance level (akin to analyzing paired data with an unpaired $t$ test; if the results were significant with an unpaired test, they would have been even more significant with a paired test).

(Manuscript submitted January 20, 2010; revision accepted for publication May 16, 2010.) 